\title{
The womb environment shapes respiratory health in offspring: a fascinating hypothesis
}

\author{
Franca Rusconi \\ Affiliation: Epidemiology Unit, Anna Meyer Children's University Hospital, Florence, Italy. \\ Correspondence: Franca Rusconi, Epidemiology Unit, Anna Meyer Children's University Hospital, Viale \\ Pieraccini 24, 50139 Florence, Italy. E-mail: franca.rusconiameyer.it
}

0 @ERSpublications

Pre-eclampsia is detrimental to respiratory health in offspring and its effect is partly mediated by prematurity http://ow.ly/kti0305B6lc

Starting with the pioneering paper published in 1990 by D.J.P. Barker, "The fetal and infant origins of adult disease: the womb may be more important than the home" [1], and continuing with more recent papers on epigenetics and transgenerational developmental programming [2], the concept that in utero environment might play an important role on future health has reached the general public [3]. As far as respiratory health is concerned, we are all aware that asthma and allergic diseases often manifest themselves in early life, and that congenital factors play an important role in these [4]. Not only have prospective birth cohort studies been instrumental to investigate in utero determinants of chronic conditions such as asthma and IgE-associated diseases [5], but also studies based on several large population registries continue to contribute to better knowledge on this field [6].

In this issue of the European Respiratory Journal, MAGnus et al. [7], in a large study based both on a national registry, comprising all births in Norway from 1996 to 2006, and on a subsample of children in the Norwegian Mother and Child Cohort Study (MoBa), examined the association between pre-eclampsia and asthma at 7 years.

Pre-eclampsia is caused by disturbed placental function and is commonly defined as de novo hypertension after 20 weeks of gestation combined with proteinuria. Pre-eclampsia is often accompanied by a fetal growth restriction or premature delivery, which is in most cases "elective" because inducing birth is the way to reverse the condition [8]. Pre-eclampsia affects between $2 \%$ and $8 \%$ of pregnancies [9] but few studies exist on the association between pre-eclampsia and several outcomes in offspring, including respiratory disorders. What we are looking for if we want to improve health of women and of their offspring, however, are not simply associations but "causal" associations [10].

\section{Pre-eclampsia and bronchopulmonary dysplasia: a causal association?}

Following this line of reasoning, in the last few years, researchers have tried to causally associate pre-eclampsia with one of the main respiratory outcomes of very pre-term birth, bronchopulmonary dysplasia (BPD) [11]. How research has evolved in this field could give us clues to understanding the possible link between pre-eclampsia and other respiratory disorders such as recurrent wheezing in infancy and asthma, and therefore it is worthwhile to mention it. First, we must acknowledge that the association between pre-eclampsia and BPD is still disputed, with a recent systematic review and meta-analysis suggesting a possible harmful effect, but with substantial heterogeneity among the studies [12]. Conversely, a causal link between this pregnancy complication and BPD has been suggested. In pre-eclampsia, two stages are known: stage 1 complicated by intrauterine growth restriction (IUGR), and stage 2 complicated

Received: Sept 092016 | Accepted after revision: Oct 272016

Conflict of interest: None declared.

Copyright @ERS 2016 
by endothelial activation and release into maternal circulation of antiangiogenic factors [8]. Antiangiogenic factors could represent the link between abnormal placentation, which is always present in pre-eclampsia, and a pathological development of lung blood vessels: this has led to the now widely accepted "vascular hypothesis" of BPD [13, 14]. Animal work has suggested that these factors disrupt lung growth [15]. IUGR, as a result of an early disturbed placental function [8], may be an additional risk factor for BPD development.

Notably, when the two main manifestations of placentation disorders (IUGR and hypertensive diseases) have been examined separately in relation with $\mathrm{BPD}$, the association for IUGR was stronger than for hypertensive disorders $[16,17]$. Being small for gestational age (SGA), a condition often overlapping with IUGR, has been associated not only with BPD in pre-term infants but also, irrespectively of being born pre-term, to poorer lung function in childhood [18], thus suggesting another "causative" link in the case of IUGR: the association of infant size and lung or airway size.

\section{Pre-eclampsia and respiratory health in preschoolers}

Do abnormal development of lung vessels, and retardation of growth in utero and at birth, in addition to early delivery, possibly also play a role in conditions like wheezing in offspring of mothers suffering from pre-eclampsia? Epidemiological, observational studies cannot give an answers but again, they can give some clues. Let us summarise here the principal published results. A recent pooled analysis of 14 European birth cohorts [19] found an association between pre-eclampsia and early wheezing, and in particular, recurrent wheezing, and this has been confirmed, though with a borderline significance, in the Avon Longitudinal Study of Parents and Children (ALSPAC) birth cohort [20]. The latter also found a weak inverse association between pre-eclampsia and forced expiratory volume in $1 \mathrm{~s}$ and maximal mid-expiratory flow, up to school age. These results are inkeeping with data on lung function in infants of a birth cohort in Australia [21] but not with the findings of a small cohort of children in Norway [22]. In children who wheeze very early in life, and in particular in those with recurrences, a congenital reduction in airway calibre/compliance seems to be a predisposing factor to wheeze $[23,24]$. As pre-eclampsia is associated with a retardation of growth in utero, it is plausible that a relationship between pre-eclampsia and recurrent wheezing in the first years of life and reduced lung function may be mediated through a detrimental effect on fetal or airway growth.

Conversely, controlling for gestational age and birth weight attenuated the associations between pre-eclampsia and wheezing and lung function in two of the previously cited studies [19, 20]. However, more than confounders, birth weight and gestational age are potential mediators, as they sit in the causal pathway between maternal pre-eclampsia and the outcomes. Adjusting for these variables would introduce a spurious association between pre-eclampsia and the outcome (so-called collider bias) if gestational age and birth weight are affected by factors that also influence the latter (e.g. congenital anomalies and smoking) [25]. The practical impossibility of taking into account all possible aspects of this web of causation makes observational studies weak (albeit the only possible) instruments for ascertaining causal relationships.

\section{Pre-eclampsia and asthma: the role of mediators and of confounders}

In this complex scenario, Magnus et al. [7] explored whether pre-term birth might mediate the association between pre-eclampsia and childhood asthma. The authors found a positive association between pre-eclampsia and asthma in the registry study, and a borderline association in the MoBa birth cohort, confirming a result found in another recent study performed in the Danish Medical Birth Registry [26] but contrary to what has been found in two other small birth cohort studies [21, 27], and in the more recent one on the ALSPAC cohort [20]. Interestingly, when MAGNus et al. [7] stratified the sample on being born pre-term, having a mother with pree-clampsia, or both, they found an increased risk of asthma at 7 years in all groups, especially in the group with both conditions. As the co-occurrence of pre-eclampsia and prematurity is clearly nonrandom, the relative effects of pre-eclampsia and prematurity and their interaction cannot be given a causal meaning (as we would do in an experiment with factorial design), but only a descriptive one. With these caveats, mediation analysis confirmed both a direct and an indirect (mediated by prematurity) effect of pre-eclampsia on childhood asthma. All these results were significant in the registry study only, where the numbers of subjects evaluated were larger than in the MoBa cohort. The large number of children in the registry made it possible to conduct also an analysis of sibling pairs discordant for asthma. The sibling design may be useful to adjust for associations due to unmeasured confounding shared by siblings, and has been used in other registries studies involving preand peri-natal putative risk factors for respiratory disorders [28]. The sibling study indicated no association between pre-eclampsia and asthma [7]. This is partially inkeeping with the previous recent study by Liu et al. [26] in the Danish Medical Birth Registry. In a case-sibling study, early-onset pre-eclampsia (thereby associated with prematurity), but not late-onset pre-eclampsia, was still associated with an increased risk of asthma, but the strength of the association was attenuated compared with the 
association obtained from the nested case-control analysis. Despite the possible limitations of the sibling study design [29], these results indicate that part of the association between pre-eclampsia and childhood asthma may be attributed to unmeasured confounding shared by siblings.

\section{What we have learned so far?}

First, we have learned that pre-eclampsia seems to be detrimental, in particular in infants born pre-term or very pre-term, for the BPD outcome, which typically develops in these infants. A potential mechanism may be the production of antiangiogenic factors, which are also markedly increased in amniotic fluid.

Second, IUGR, being SGA and prematurity, which are all part of the clinical picture of pre-eclampsia [8], have been found to be associated with wheezing up to school age [30,31]. An arrested growth of the lung in utero and a resulting diminished calibre of the airways are likely candidates explaining the association of pre-eclampsia per se and wheezing and diminished lung function.

Finally, whatever the mechanisms involved and independently of direct versus indirect effect of pre-eclampsia on respiratory disorders in offspring, these results are robust in describing an increased risk of respiratory problems in offspring of pre-eclamptic mothers.

Thus, what counts from the point of view of public health is firstly, to control the disease, which still has a high prevalence in low-income countries and in low socioeconomic classes [8], and is a major contributor to maternal mortality, and secondly, to be aware that offspring of pre-eclamptic mothers are at risk of adverse respiratory outcomes and deserve a careful clinical follow-up.

\section{References}

1 Barker DJP. The fetal and infant origins of adult disease: the womb may be more important than the home. BMJ 1990; 301: 1111.

2 Aiken CE, Ozanne SE. Transgenerational developmental programming. Hum Reprod Update 2014; 20: 63-75.

3 Murphy Paul A. How the first nine months shape the rest of your life - the new science of fetal origins. Time 2010; 176: 51-55.

4 Duijts L. Fetal and infant origins of asthma. Eur J Epidemiol 2012; 27: 5-14.

5 Bousquet J, Anto J, Sunyer J, et al. Pooling birth cohorts in allergy and asthma: European Union-funded initiatives a, CHICOS, ENRIECO, and GA ${ }^{2}$ LEN joint paper. Int Arch Allergy Immunol 2013; 161: 1-10.

6 Thomsen SF. Exploring the origins of asthma: Lessons from twin studies. Eur Clin Respir J 2014; 1: Suppl. 1, 25535.

7 Magnus MC, Håberg SE, Magnus P, et al. Pre-eclampsia and childhood asthma. Eur Respir J 2016; 48: 1622-1630. Steegers EA, von Dadelszen P, Duvekot JJ, et al. Pre-eclampsia. Lancet 2010; 376: 631-644.

9 Roberts CL, Ford JB, Algert CS, et al. Population-based trends in pregnancy hypertension and pre-eclampsia: an international comparative study. BMJ Open 2011; 1: e000101.

10 Gagliardi L. Prediction and causal inference. Acta Paediatr 2009; 98: 1890-1892.

11 Hansen AR, Barnés CM, Folkman J, et al. Maternal preeclampsia predicts the development of bronchopulmonary dysplasia. J Pediatr 2010; 156: 532-536.

$12 \mathrm{Bi}$ GL, Chen FL, Huang WM. The association between hypertensive disorders in pregnancy and bronchopulmonary dysplasia: a systematic review. World J Pediatr 2013; 9: 300-306.

13 Levine RJ, Maynard SE, Qian C, et al. Circulating angiogenic factors and the risk of preeclampsia. N Engl J Med 2004; 350: 672-683.

14 Thebaud B, Lacaze-Malmonteil T. If your placenta doesn't have it, chances are your lungs don't have it either: the "vascular hypothesis" of bronchopulmonary dysplasia starts in utero. J Pediatr 2010; 156: 521-523.

15 Tang JR, Karumanchi SA, Seedorf G, et al. Excess soluble vascular endothelial growth factor receptor-1 in amniotic fluid impairs lung growth in rats: Linking preeclampsia with bronchopulmonary dysplasia. Am J Physiol Lung Cell Mol Physiol 2012; 302: L36-L46.

16 Gagliardi L, Rusconi F, Da Frè M, et al. Pregnancy disorders leading to very preterm birth influence neonatal outcomes: results of the population-based ACTION cohort study. Pediatr Res 2013; 73: 794-801.

17 Torchin H, Ancel PY, Goffinet F, et al. Placental complications and bronchopulmonary dysplasia: EPIPAGE-2 cohort study. Pediatrics 2016; 137: e20152163.

18 Kotecha SJ, Watkins WJ, Heron J, et al. Spirometric lung function in school-age children. Effect of Intrauterine growth retardation and catch-up growth. Am J Respir Crit Care Med 2010; 181: 969-974.

19 Zugna D, Galassi C, Annesi-Maesano I, et al. Maternal complications in pregnancy and wheezing in early childhood: a pooled analysis of 14 birth cohorts. Int J Epidemiol 2015; 44: 199-208.

20 Shaheen SO, Macdonald-Wallis C, Lawlor DA, et al. Hypertensive disorders of pregnancy, respiratory outcomes and atopy in childhood. Eur Respir J 2016; 47: 156-165.

21 Stick SM, Burton PR, Gurrin L, et al. Effects of maternal smoking during pregnancy and a family history of asthma on respiratory function in newborn infants. Lancet 1996; 348: 1060-1064.

22 Byberg KK, Ogland B, Eide GE, et al. Birth after preeclamptic pregnancies: association with allergic sensitization and allergic rhinoconjunctivitis in late childhood; a historically matched cohort study. BMC Pediatr 2014; 14: 101.

23 Martinez FD, Morgan WJ, Wright AL, et al. Diminished lung function as a predisposing factor for wheezing respiratory illness in infants. N Engl J Med 1988; 319: 1112-1117.

24 Young S, Arnott J, O'Keeffe PT, et al. The association between early life lung function and wheezing during the first 2 yrs of life. Eur Respir J 2000; 15: 151-157.

25 Wilcox AJ, Weinberg CR, Basso O. On the pitfalls of adjusting for gestational age at birth. Am J Epidemiol 2011; 174: 1062-1068. 
26 Liu X, Olsen J, Agerbo E, et al. Maternal preeclampsia and childhood asthma in the offspring. Pediatr Allergy Immunol 2015; 26: 181-185.

27 Nafstad P, Samuelsen SO, Irgens LM, et al. Pregnancy complications and the risk of asthma among Norwegians born between 1967 and 1993. Eur J Epidemiol 2003; 18: 755-761.

28 Örtqvist AK, Lundholm C, Kieler H, et al. Antibiotics in fetal and early life and subsequent childhood asthma: nationwide population based study with sibling analysis. BMJ 2014; 349: g6979.

29 Frisell T, Öberg S, Kuja-Halkola R, et al. Sibling comparison designs. Epidemiology 2012; 23: 713-720.

30 Been JV, Lugtenberg MJ, Smets E, et al. Preterm birth and childhood wheezing disorders: a systematic review and meta-analysis. PLoS Med 2014; 11: e1001596.

31 Mebrahtu TF, Feltbower RG, Greenwood DC, et al. Birth weight and childhood wheezing disorders: a systematic review and meta-analysis. J Epidemiol Community Health 2015; 69: 500-508. 